\title{
Polyphenol-rich diets improve glucose metabolism in people at high cardiometabolic risk: a controlled randomised intervention trial
}

\author{
Lutgarda Bozzetto $^{1} \cdot$ Giovanni Annuzzi $^{1}$ - Giovanni Pacini ${ }^{2} \cdot$ Giuseppina Costabile $^{1}$. \\ Claudia Vetrani ${ }^{1} \cdot$ Marilena Vitale $^{1} \cdot$ Ettore Griffo $^{1} \cdot$ Angela Giacco $^{1} \cdot$ \\ Claudia De Natale ${ }^{1} \cdot$ Sara Cocozza $^{1}$ - Giuseppe Della Pepa ${ }^{1}$ - Andrea Tura ${ }^{2}$. \\ Gabriele Riccardi $^{1} \cdot$ Angela A. Rivellese $^{1}$
}

Received: 28 December 2014 / Accepted: 23 March 2015 /Published online: 24 April 2015

(C) Springer-Verlag Berlin Heidelberg 2015

\begin{abstract}
Aim/hypothesis Dietary polyphenols and long chain $n-3$ polyunsaturated fatty acids ( $\mathrm{LCn} 3)$ are associated with lower cardiovascular risk. This may relate to their influence on glucose metabolism and diabetes risk. We evaluated the effects of diets naturally rich in polyphenols and/or LCn3 of marine origin on glucose metabolism in people at high cardiometabolic risk. Methods According to a $2 \times 2$ factorial design, individuals with high waist circumference and at least one more component of the metabolic syndrome were recruited at the obesity outpatient clinic. Eighty-six participants were randomly assigned by MINIM software to an isoenergetic diet: (1) control, low in LCn3 and polyphenol (analysed $n=20$ ); (2) rich in LCn3 ( $n=19)$; (3) rich in polyphenols $(n=19)$; or (4) rich in LCn3 and polyphenols $(n=19)$. The assigned diets were known for the participants and blinded for people doing measurements. Before and after the 8 week intervention, participants underwent a $3 \mathrm{~h}$ OGTT and a test meal with a similar composition as the assigned diet for the evaluation of plasma glucose, insulin and glucagon-like peptide 1 (GLP-1)
\end{abstract}

Electronic supplementary material The online version of this article (doi:10.1007/s00125-015-3592-x) contains peer-reviewed but unedited supplementary material, which is available to authorised users.

Angela A. Rivellese

rivelles@unina.it

1 Department of Clinical Medicine and Surgery, Federico II University, Via Pansini, 5, 80131 Naples, Italy

2 Metabolic Unit, Institute of Biomedical Engineering, National Research Council, Padua, Italy concentrations, and indices of insulin sensitivity and beta cell function.

Results During OGTT, polyphenols significantly reduced plasma glucose total AUC $(p=0.038)$ and increased early insulin secretion $(p=0.048)$, while LCn3 significantly reduced beta cell function $(p=0.031)$ (two-factor ANOVA). Moreover, polyphenols improved post-challenge oral glucose insulin sensitivity (OGIS; $p=0.05$ vs control diet by post hoc ANOVA). At test meal, LCn3 significantly reduced GLP-1 total postprandial AUC ( $p<0.001$; two-factor ANOVA).

Conclusion/interpretation Diets naturally rich in polyphenols reduce blood glucose response, likely by increasing early insulin secretion and insulin sensitivity. These effects may favourably influence diabetes and cardiovascular risk. The implications of the decrease in insulin secretion and postprandial GLP-1 observed with diets rich in marine LCn3 need further clarification.

Trial registration: ClinicalTrials.gov NCT01154478

Funding: The trial was funded by European Community's Seventh Framework Programme FP7/2009-2012 under grant agreement FP7-KBBE-222639, Etherpaths Project and 'Ministero Istruzione Università e Ricerca' PRIN 2010-2011 - 2010JCWWKM.

Keywords Beta cell function · Diet · GLP-1 - Glucose metabolism · Insulin secretion · Insulin sensitivity $\cdot$ Long chain $n-3$ polyunsaturated fatty acids $\cdot$ OGTT $\cdot$ Oral glucose insulin sensitivity $\cdot$ Polyphenols
Abbreviations
CVD
Cardiovascular disease
GLP-1
Glucagon-like peptide-1 


High LCn3
High LCn3\&PP
High PP
HOMA-B
IFG
IGT
LCn3
OGIS
QUICKI

High LCn3 diet

High LCn3 and polyphenol diet

High polyphenol diet

HOMA for beta cell function

Impaired fasting glucose

Impaired glucose tolerance

Long chain $n-3$ polyunsaturated fatty

acids

Oral glucose insulin sensitivity

Quantitative insulin sensitivity check

index

\section{Introduction}

Dietary polyphenols and long chain $n-3$ polyunsaturated fatty acids (LCn3) are associated with lower cardiovascular disease (CVD) risk [1,2]. Alterations of glucose metabolism are involved in the pathogenesis of CVD [3]; therefore, diets rich in polyphenols and/or LCn3 may have a favourable impact on CVD also by improving glucose metabolism.

Several epidemiological studies have shown that dietary intake of polyphenols was inversely related to risk of type 2 diabetes [4-6]. Numerous in vitro and animal studies also support this finding, reporting beneficial effects of dietary polyphenols on carbohydrate digestion, and insulin sensitivity and secretion through the activation of various intracellular molecular pathways [7]. Few clinical trials have explored this issue in humans, mostly confirming beneficial effects of either supplementation with extracts from foods rich in polyphenols [8], or regular consumption for a short period of single foods (green tea, chocolate, red wine etc.) $[9,10]$. Therefore, information about the efficacy of nutritional interventions, reproducing real-life conditions, based on various natural foods rich in polyphenols is lacking. This evidence would be relevant because a holistic dietary approach including a huge variety of food choices would be feasible and therefore applicable in practical care. Moreover, because polyphenols differ in terms of bioavailability and intermediate metabolism, the simultaneous intake of different classes of these compounds could differently influence glucose metabolism compared with the intake of single supplements or foods leading to an enhanced clinical effect.

Several trials have explored the effects of short- and medium-term LCn3 supplementation on surrogate markers of type 2 diabetes risk, i.e. insulin sensitivity, insulin secretion, fasting blood glucose concentrations in different populations, and have found beneficial, detrimental or no effects [11-15]. Only a few studies have evaluated the effects on glucose metabolism of LCn3 derived from dietary fish intake. They showed an improvement in insulin sensitivity when fish consumption was associated with calorie restriction [16] or physical training [17], while no effect on glucose metabolism was

observed following an isocaloric dietary intervention with fish [18].

Against this background, the aim of our randomised clinical trial was to explore, in individuals at risk of type 2 diabetes, the medium-term effects of diets naturally rich in different sources of polyphenols and/or marine LCn3s on glucose metabolism.

\section{Methods}

\section{Participants}

Eighty-six individuals of both sexes, aged 35-70 years, with overweight or obesity (BMI $27-35 \mathrm{~kg} / \mathrm{m}^{2}$ ), high waist circumference (men $>102 \mathrm{~cm}$ and women $>88 \mathrm{~cm}$ ) and meeting at least one or more criteria for the metabolic syndrome diagnosis according to the National Cholesterol Education Program (NCEP)/Adult Treatment Program (ATP) III [19] were recruited at the obesity outpatient clinic of the Federico II University Hospital (Naples, Italy). Exclusion criteria were: fasting plasma triacylglycerols $\geq 4.5 \mathrm{mmol} / 1$, fasting cholesterol $>7 \mathrm{mmol} / \mathrm{l}$, cardiovascular events (myocardial attack or stroke) during the 6 months prior to the study, established diabetes mellitus, regular intensive exercise, renal and liver failure, or any other chronic disease, or use of drugs able to influence inflammation, and lipid and glucose metabolism. The participants had stable food habits, were not vegetarians and were asked to refrain from any dietary supplement for 1 month prior to and during the study. Inclusion and exclusion criteria were assessed by interviews, clinical examination and routine laboratory tests. Diabetes status was excluded by a 75 g OGTT.

Eight participants, equally distributed in the four experimental groups, did not undergo the final test for work- or family-related reasons. The main clinical characteristics of the 78 participants who completed the dietary intervention are shown in Table 1.

The design of the trial was approved by the Federico II University Ethics Committee, complied with the Helsinki Declaration guidelines, and was registered at ClinicalTrials.gov registration number NCT01154478. All participants provided written informed consent. The results of this dietary intervention trial on lipid metabolism have been previously published [20], and this previous publication includes a participant flow chart.

\section{Study design}

During a 3 week run-in period the participants were stabilised on their habitual diet. Their isoenergetic requirements were determined taking into account the individual's habitual energy intake, evaluated by a 7 day food record, adjusted for body weight and the dietitian's clinical evaluation. Thereafter, they 
Table 1 Baseline characteristics of the participants of the four diet groups

\begin{tabular}{llllll}
\hline & Control & High LCn3 & High PP & High LCn3\&PP & $p$ value (ANOVA) \\
\hline Sex (M/F) & $8 / 12$ & $8 / 11$ & $9 / 11$ & $8 / 11$ & 0.645 \\
Age (years) & $54 \pm 9$ & $56 \pm 8$ & $53 \pm 9$ & $55 \pm 9$ & 0.858 \\
Body weight (kg) & $87 \pm 10$ & $86 \pm 12$ & $87 \pm 11$ & $84 \pm 12$ & 0.126 \\
BMI (kg/m ${ }^{2}$ ) & $33 \pm 3$ & $32 \pm 4$ & $32 \pm 3$ & $30 \pm 3$ & 0.601 \\
Waist circumference (cm) & $104 \pm 7$ & $105 \pm 10$ & $104 \pm 9$ & $101 \pm 8$ & 0.231 \\
Systolic BP (mmHg) & $120 \pm 7$ & $121 \pm 12$ & $126 \pm 16$ & $119 \pm 9$ & 0.663 \\
Diastolic BP (mmHg) & $76 \pm 8$ & $74 \pm 7$ & $76 \pm 9$ & $73 \pm 8$ & 0.498 \\
Fasting plasma glucose (mmol/l) & $5.75 \pm 0.7$ & $5.83 \pm 0.6$ & $5.57 \pm 0.5$ & $5.74 \pm 0.6$ & 0.220 \\
Fasting plasma insulin (pmol/l) & $118 \pm 38$ & $140 \pm 50$ & $142 \pm 44$ & $120 \pm 45$ & 0.960 \\
GLP-1 (pmol/l) & $3.49 \pm 1.40$ & $3.94 \pm 2.21$ & $3.78 \pm 2.77$ & $3.72 \pm 3.63$ & 0.398 \\
QUICKI & $0.37 \pm 0.03$ & $0.36 \pm 0.02$ & $0.36 \pm 0.02$ & $0.37 \pm 0.02$ & \\
\hline
\end{tabular}

Data are means \pm SD

were randomised to follow an 8 week experimental diet according to a $2 \times 2$ factorial design, as previously described in detail [20]. Patients were aware of which diet group they had been assigned to. Diets were isoenergetic and only differed for the amount of polyphenol and LCn3: (1) Control diet (Control), low in LCn3 and polyphenols (1.5 g and $365 \mathrm{mg} /$ day, respectively); (2) High LCn3 diet (High LCn3), rich in LCn3 and low in polyphenols ( $4 \mathrm{~g}$ and $363 \mathrm{mg} /$ day, respectively); (3) High polyphenol diet (High PP), low in LCn3 and rich in polyphenols (1.4 g and 2,903 mg/day, respectively); (4) High LCn3 and high polyphenol diet (High LCn3\&PP), rich in $\mathrm{LCn} 3$ and polyphenols (4 $\mathrm{g}$ and $2,861 \mathrm{mg}$ /day, respectively). They were, instead, similar for other macronutrients and micronutrients as reported in our previous paper [20]. Similar amounts of olive oil were used as seasoning in all diets, with the only difference being that extra-virgin olive oil was used in the polyphenol-rich diets and non-extra-virgin olive oil in the polyphenol-poor diets. Therefore, the diets differed for the polyphenol content of olive oil (higher in the extra-virgin olive oil) and not for the other olive oil components.

The main dietary sources of LCn 3 were salmon, dentex and anchovies. Polyphenols were supplied by decaffeinated green tea and coffee, dark chocolate, blueberry jam, extra-virgin olive oil and polyphenol-rich vegetables (artichokes, onions, spinach and rocket). Rocket was consumed raw, while the other vegetables were steamed, in order to reduce the effect of the cooking process on polyphenol availability [21]. The main polyphenol classes were flavonoids (57\%) - mainly represented by flavanols (41\%) - and phenolic acids (43\%). The polyphenol content of foods was measured by the FolinCiocalteu method [22] or calculated according to US Department of Agriculture (USDA) tables [23], and LCn3 levels were calculated from the Italian Food Composition Database [24]. No alcohol was allowed during the study. To improve dietary compliance, all meals and beverages were provided to the participants weekly for the whole study period. Meals were prepared under the supervision of an expert dietitian. The participants received dietary counselling every week and phone calls every 2-3 days. Adherence to diet was evaluated by a 7 day food record performed at baseline, 4 weeks and 8 weeks.

The participants were instructed to not change their physical activity and smoking habits during the study.

\section{Experimental procedures and laboratory methods}

At baseline and after the 8 week intervention, body weight, height and waist circumference were measured according to standardised procedures. After a $12 \mathrm{~h}$ overnight fast, the participants underwent a $75 \mathrm{~g}$ OGTT, with blood sampling at 0 , $15,30,60,90,120,150$ and $180 \mathrm{~min}$. Two days later, after a $12 \mathrm{~h}$ overnight fast, they underwent a test meal rich in fat with blood sampling at $0,60,120,240$ and $360 \mathrm{~min}$ for the evaluation of postprandial lipaemia. Meals were composed of rice, butter, parmesan cheese, bresaola and white bread, with intakes of olive oil, salmon and decaffeinated green tea differing in order to obtain a similar composition as the assigned diet [20]. Blood drawn in EDTA- or EDTA and aprotinin (for the glucagon-like peptide 1 [GLP-1] assay) tubes was centrifuged and plasma stored at $-80^{\circ} \mathrm{C}$ until measurement. Glucose concentrations were assayed by enzymatic colorimetric methods (ABX Diagnostics, Montpellier, France) on a Cobas Mira Autoanalyzer (ABX Diagnostics). Insulin concentrations were measured by ELISA (DIAsource ImmunoAssays, Nivelles, Belgium) on a Triturus Analyzer (Diagnostics Grifols, Barcelona, Spain). Active GLP-1 was assayed by a nonradioactive, highly specific sandwich ELISA method (MerckMillipore, Darmstadt, Germany) that had $100 \%$ crossreactivity with active isoforms of GLP-1 (7-36 amide and 7-37 glycine extended) but no reactivity with inactive isoforms (9-36 amide and 9-37 glycine extended), GLP-2 or 
glucagon [25]. Laboratory analyses were performed blinded to the assigned treatment.

\section{Calculations}

Fasting insulin sensitivity was evaluated using the quantitative insulin sensitivity check index (QUICKI) [26]. Fasting insulin secretion capacity was calculated as the HOMA for beta cell function [HOMA-B: (20 fasting insulin, pmol/l)/(fasting glucose, $\mathrm{mmol} / \mathrm{l}-3.5)]$ [27].

During OGTT and test meal, the total AUC was calculated by the trapezoidal method.

After glucose load, insulin action was evaluated by the 180 min oral glucose insulin sensitivity (OGIS) method [26] and insulin secretion capacity was calculated as beta cell function [insulin 0-180 AUC/glucose 0-180 AUC ratio] [26] and early phase insulin secretion (insulin 0-30 AUC).

\section{Statistical analysis}

The sample size ( 80 patients) was calculated on the primary outcome, i.e. postprandial lipid response [20]. This sample size was also adequate to detect a $0.3 \mathrm{mmol} / \mathrm{h}$ reduction in post-load blood glucose AUC that corresponds to the difference previously observed in post-load glucose AUC between healthy people and individuals at high risk of diabetes [27]. We sought to see this difference with an $80 \%$ power at the $5 \%$ significance level. To take care of a possible dropout, 86 participants were enrolled. The random allocation to the intervention, stratified for sex, age, BMI and plasma triacylglycerols was performed by the minimisation method using MINIM software, version 1.5 (www-users.york.ac.uk/ mb55/guide/minim.htm).

Data were expressed as means \pm SD unless otherwise stated. Variables not normally distributed were analysed after logarithmic transformation $\left(\log _{10}\right)$. The differences in baseline characteristics between the four groups were analysed by ANOVA and post hoc analysis (least significant difference [LSD] test). The effects on absolute changes ( 8 weeks minus baseline) of dietary polyphenols, dietary $\mathrm{LCn} 3$, and the interaction between polyphenols and LCn3 were evaluated by two-factor ANOVA adjusting for baseline values. The effects of dietary treatments on post-challenge profiles (OGTT, test meal) were evaluated by general linear model for repeated measures.

Statistical analysis was performed according to standard methods using the Statistical Package for Social Sciences software 21.0 (SPSS/PC; SPSS, Chicago, IL, USA).

\section{Results}

Seventy-eight participants completed the trial (Control, $n=20$; High LCn3, $n=19$; High PP, $n=20$; High LCn3\&PP, $n=19$ ).
Data of the final OGTT were not available for one individual in the High PP group owing to technical problems.

At baseline, participants allocated to the four intervention groups were comparable for age, body weight, BMI, BP, fasting plasma concentrations of lipids, glucose, insulin and GLP-1, and fasting insulin sensitivity calculated with QUICKI (Table 1).

At baseline, 25 participants had impaired fasting glucose (IFG; Control, $n=5$; High LCn3, $n=6$; High PP, $n=6$; High PP\&LCn3, $n=8$ ); eight participants had impaired glucose tolerance (IGT; Control, $n=1$; High LCn3, $n=4$; High PP, $n=2$; High PP\&LCn3, $n=1$ ); and 18 participants had IFG+IGT (Control, $n=4$; High LCn3, $n=5$; High PP, $n=7$; High PP\&LCn3, $n=2$ ).

\section{Compliance to dietary intervention and anthropometrics measures}

As previously reported [20], compliance to the experimental diets was optimal in all groups. In fact, all participants were within the ranges of intakes defined for good compliance ( $\pm 20 \%$ of assigned intake).

Body weight did not change after the intervention except for a small but statistically significant reduction in the High LCn3 group $(-1.14 \pm 1.3 \mathrm{~kg})$ compared with baseline $(p=$ 0.041 ); waist circumference did not change significantly during the intervention [20].

\section{Fasting indices of glucose metabolism}

The 8 week intervention with polyphenols, LCn3 or their combination did not induce any significant change in fasting plasma concentrations of glucose and insulin, and fasting indices of insulin sensitivity and insulin secretion capacity (Table 2).

\section{Glucose, insulin and derived indices during OGTT}

Glucose and insulin concentrations The absolute changes after the intervention ( 8 week minus baseline) in OGTT plasma glucose and insulin mean values are reported either as single time points (Fig. 1a and c) or as AUC (Fig. 1b and d). Individual changes in glucose and insulin AUCs are reported in electronic supplementary material (ESM) Fig. 1. Polyphenols significantly decreased the glycaemic response to glucose load as shown by absolute changes in total blood glucose AUC (Control, 1.67 \pm 3.56 ; High LCn3, 0.94 \pm 3.56 ; High PP, $-0.89 \pm 3.56$; High LCn3\&PP, $0.17 \pm 4.11 \mathrm{mmol} / 13 \mathrm{~h}$; mean \pm $\mathrm{SD} ; p=0.036$ for polyphenol effect by two-factor ANOVA), whereas no significant effect for LCn3s or their interaction was found. The glucose lowering effect of the polyphenol enriched diet was more marked in the second part of the 


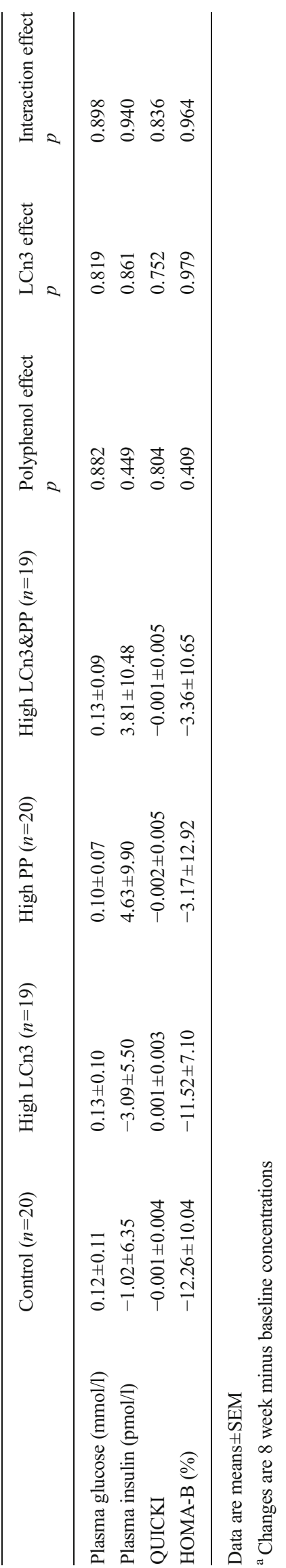


Fig. 1 (a-d) Absolute changes after dietary intervention ( 8 week minus baseline) of plasma glucose and insulin concentrations at each time point $(\mathbf{a}, \mathbf{c})$ and total AUC (b, d) during OGTT. Black squares, Control diet; white squares, High LCn3; black triangles, High PP; white triangles, High LCn3\&PP. Data are means \pm SEM. ${ }^{*} p<0.05$ for polyphenol effect by two-factor ANOVA at single time points; ${ }^{\dagger} p=0.036$ for polyphenol effect by two-factor ANOVA. Ctrl, Control diet; LCn3, High LCn3; PP, High PP; LCn3\&PP, High LCn3\&PP a

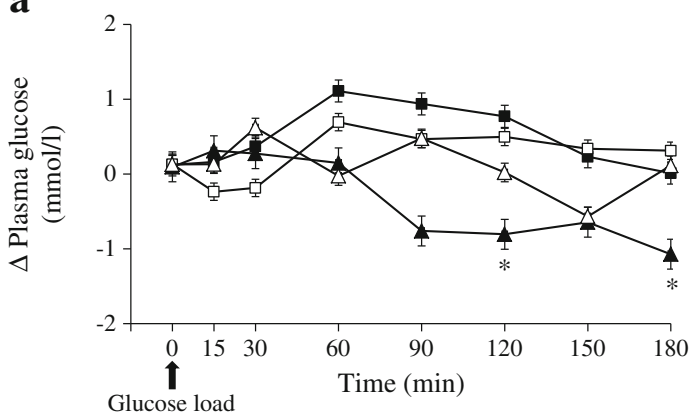

c

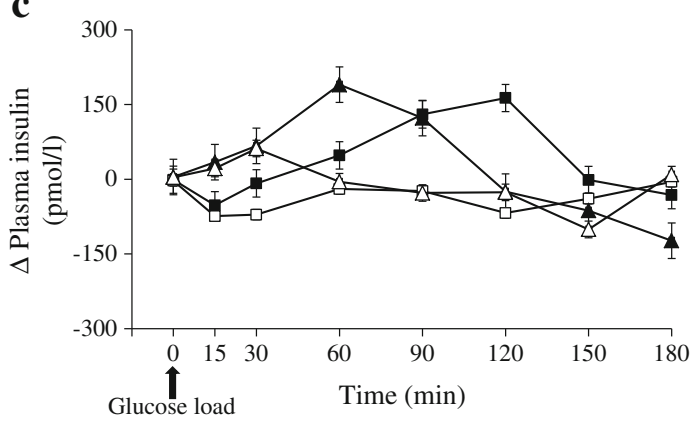

b

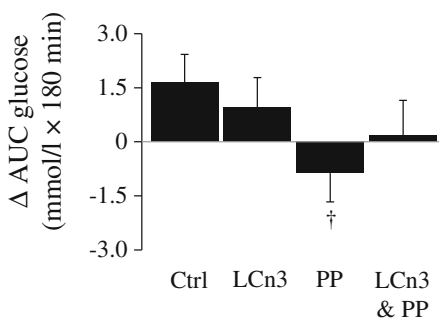

d

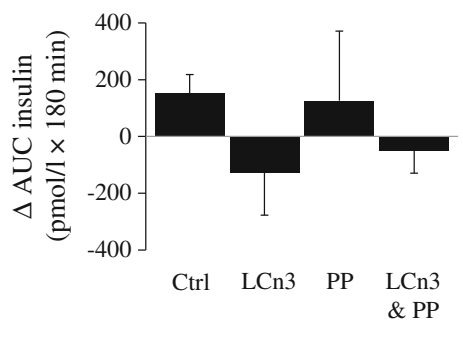

OGTT $(90-120 \mathrm{~min} ; p=0.025$ for the time $\times$ polyphenol interaction by general linear model for repeated measures).

Figure 1c, d reports the results of plasma insulin during OGTT: no significant difference in total plasma insulin responses (AUC 0-180 min) was observed among the different diets.

Insulin secretion The early phase of insulin secretion (AUC 0-30 min; Fig. 2a) was significantly increased in the High PP groups ( $p=0.048$ by two-factor ANOVA) while there was no effect for $\mathrm{LCn} 3 \mathrm{~s}$ or their interactions.

Beta cell function Absolute changes in beta cell function are shown in Fig. 2b. This index of insulin secretion was increased in the High PP group and decreased in the High LCn3 and High LCn3\&PP groups. By two-factor ANOVA, only the effect of LCn3 was significant ( $p=0.031$ ), whereas no significant effects for polyphenols or their interactions were found.

Insulin sensitivity Insulin sensitivity during OGTT evaluated by OGIS was not significantly different among the dietary intervention groups according to two-factor ANOVA. By comparing each group with the control, the High PP group had a significantly higher increase in insulin sensitivity than the Control diet group (Fig. 2c; $p=0.050$ vs Control diet by post hoc ANOVA).

\section{Glucose, insulin and GLP-1 during the test meal}

The absolute changes after the intervention ( 8 week minus baseline) in plasma glucose, insulin and GLP-1 during the test meal are reported in Fig. 3, either as single time points or AUC. No significant differences in plasma glucose postprandial AUCs were observed. However, postprandial plasma glucose concentrations tended to increase after the $\mathrm{LCn} 3$ rich diets reaching statistical significance at $120 \mathrm{~min}$ (Fig. 3a). Postprandial insulin AUC showed a non-significant increase after High LCn3\&PP and non-significant decreases after both High LCn3 and High PP (Fig. 3d). High LCn3 decreased postprandial plasma GLP-1 concentrations with significant differences in absolute changes at 30, 60, 120 and $180 \mathrm{~min}$ time points (Fig. 3e) and total AUC (Control, 122 \pm 351 ; High LCn3, -147 \pm 206 ; High PP, $-15 \pm 289$; High LCn3\&PP, -239 $\pm 322 \mathrm{pmol} / 13 \mathrm{~h} ; p<0.0001$ for LCn3 effect by two-factor ANOVA; Fig. 3f). No significant effect for polyphenols or their interaction was found (Fig. $3 \mathrm{f}$ and e).

\section{Discussion}

The main and most relevant finding of our study was that diets naturally rich in polyphenols reduced plasma glucose concentrations and increased the early phase of insulin secretion in response to an OGTT. Moreover, with the polyphenol-rich diet, an improvement of post-challenge insulin sensitivity was also observed.

Recent intervention studies showed that the adhesion to a healthy Nordic diet [28] has some beneficial effects on glucose metabolism and that the Mediterranean diet [29] reduces the incidence of CVD and prevents type 2 diabetes. These beneficial dietary patterns consisted of multiple dietary 

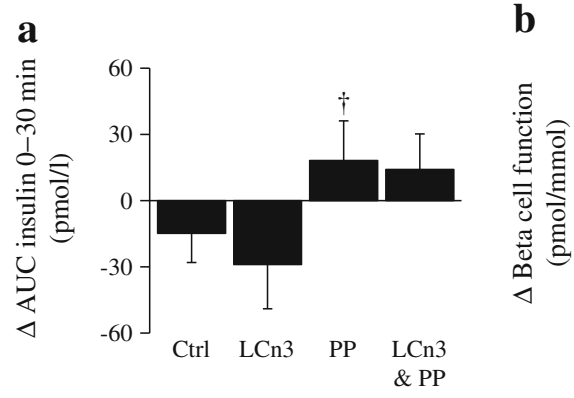

Fig. 2 (a-c) Absolute changes after dietary intervention (8 week minus baseline) of plasma insulin 0-30 min AUC (a), beta cell function (b) and OGIS (c) during OGTT. Data are means \pm SEM. ${ }^{\dagger} p=0.048$ for polyphenol

changes with potential favourable effects on glucose metabolism and cardiovascular risk factors, such as a reduction in saturated fat, an increase in monounsaturated and polyunsaturated fatty acids, fibre and polyphenols. Therefore, these experimental approaches, although giving clinically relevant evidence, do not provide information on how and to what extent each dietary component beneficially acts. The present study shows that a diet naturally rich in polyphenols deriving from different foods and beverages, independently of the other dietary components, has favourable effects on glucose c

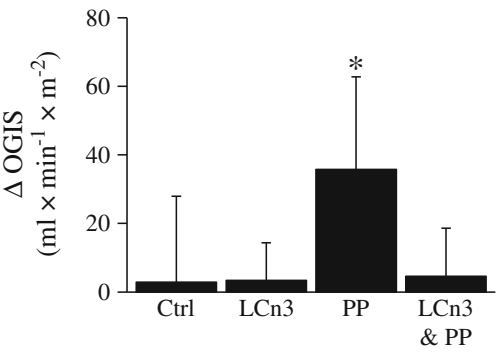

effect by two-factor ANOVA; ${ }^{\star} p=0.031$ for LCn3 effect by two-factor ANOVA. ${ }^{*} p=0.05$ vs the control diet by post hoc ANOVA. Ctrl, Control diet; LCn3, High LCn3; PP, High PP; LCn3\&PP, High LCn3\&PP

metabolism in people at high risk of diabetes, suggesting that the metabolic benefits associated with some dietary patterns may be due, among other factors, to their polyphenol content.

Our results show an improvement in glucose metabolism during an OGTT and therefore in the absence of an acute intake of polyphenols. This suggests that the chronic intake of polyphenols induces long lasting modifications of the metabolic and genetic pathways involved in the regulation of glucose metabolism. In particular, the decrease in plasma glucose concentrations observed during the OGTT was

Fig. 3 (a-f) Absolute changes after dietary intervention ( 8 week minus baseline) of plasma glucose $(\mathbf{a}, \mathbf{b})$, insulin $(\mathbf{c}, \mathbf{d})$ and GLP-1 (e, f) concentrations at single time points $(\mathbf{a}, \mathbf{c}, \mathbf{e})$ and total AUC (b, d, f) after test meal. Black squares, Control diet; white squares, High LCn3; black triangles, High PP; white triangles, High LCn3\&PP. Data are means $\pm \mathrm{SEM} ;{ }^{*} p<0.05$ for $\mathrm{LCn} 3$ effect by two-factor ANOVA at single time points. ${ }^{\dagger} p=0.0001$ for LCn3 effect by two-factor ANOVA. Ctrl, Control diet; LCn3, High LCn3; PP, High PP; LCn3\&PP, High LCn3 \& PP

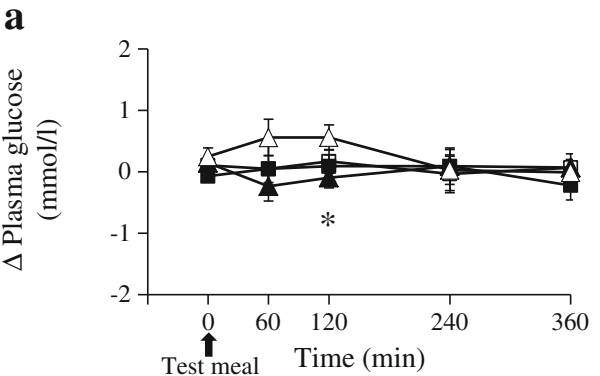

c
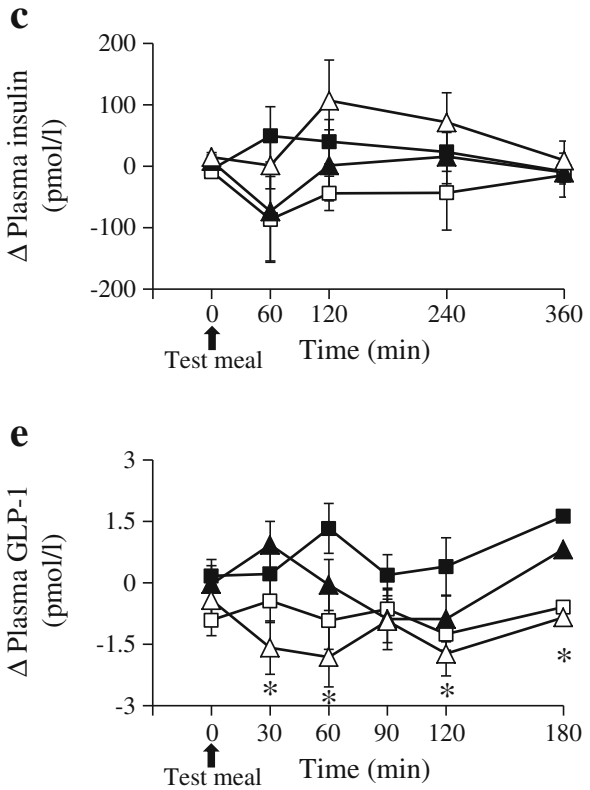

b

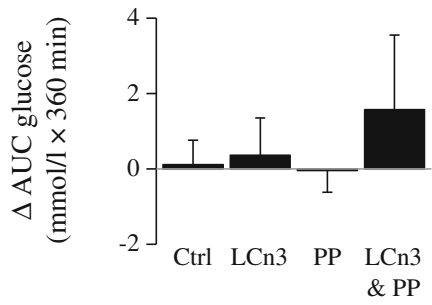

d
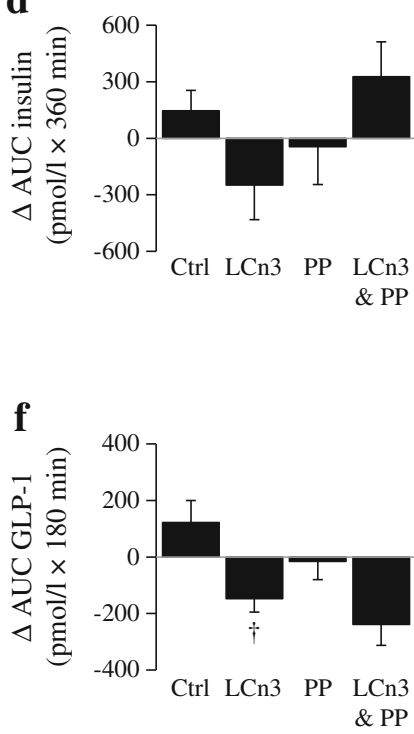
associated with an improvement of the first-phase of insulin secretion. Moreover, as shown by OGTT-related indices, dietary polyphenols tended to improve also post-challenge insulin sensitivity. Evidence from several in vitro and animal studies supports the mechanisms of beneficial action of polyphenols on glucose metabolism observed in our study. In different experimental settings, various polyphenol classes were able to enhance insulin secretion both through glucose dependent [30] and independent [31] mechanisms and by stimulating beta cell proliferation [32].

Some studies have also shown that polyphenols might improve glucose uptake from peripheral tissues directly influencing the action of key regulating genes such as peroxisome proliferator-activated receptor $\gamma$ (PPAR- $\gamma$ ) and the translocation of the glucose-transporter GLUT-4 as shown in cultured adipocytes [33].

In our intervention, the use of different classes of polyphenols, each with a possible different mechanism of action on glucose metabolism, may explain the simultaneous influence on different mechanisms, first-phase insulin secretion and insulin sensitivity with a subsequent additive and clearly beneficial effect on glucose tolerance.

The beneficial effects of diets higher in polyphenols on glucose metabolism observed during OGTT were not evident after a meal with the same composition as the followed diet. It is possible that the test meals-which were poor in carbohydrate and rich in fat in order to evaluate an adequate postprandial lipid response - were not optimal to elicit differences in postprandial glucose response.

We also observed that diets with higher amounts of fish, rich in LCn3, reduced beta cell function as measured by OGTT-derived indices, without influencing insulin sensitivity. This was in line with the tendency to higher glucose and lower insulin levels after the test meal. These lower insulin levels were not evident after the combined High LCn3\&PP diet, suggesting that polyphenols intake may blunt the deleterious effect on insulin secretion of LCn3.

Previous studies with different experimental designs explored the effects of $\mathrm{LCn} 3$ on glucose metabolism giving conflicting results [13, 34-36]. Therefore, the pathophysiological interpretation of our findings on insulin secretion is not univocal as it might be both the consequence of lipotoxicity due to the exposure to high concentrations of fatty acids, in particular LCn3, as shown by in vitro studies [36, 37], but also a physiological mechanism of preservation of beta cell function [36]. In this respect, an interesting finding of our study is that, in line with data from an acute study [38], the dietary intervention with LCn3 reduced the postprandial GLP-1 response. It is, therefore, possible that this effect of $\mathrm{LCn} 3$ observed after the test meal may be related to the decrease in beta cell function found after the glucose challenge.

As for clinical implications, the results of this study are relevant as they show, for the first time, that a diet containing foods rich in polyphenols may beneficially influence glucose metabolism in individuals at high risk of diabetes. Therefore, an increase in the intake of foods naturally rich in polyphenols should be recommended in these individuals to improve their cardiometabolic profile. The amount of polyphenols used in our study was higher compared with the amount generally consumed in western diets ( $1 \mathrm{~g} /$ day) [39] but is easily reached with natural foods typical of our as well other gastronomic backgrounds and without any kind of supplement. Moreover, the very good adherence of participants shows that this type of diet is feasible and easily acceptable. A further strength of our controlled study was that the experimental diets differed in particular in the two components that we aimed to evaluate, i.e. polyphenols and LCn3. Therefore, we can state that, to the best of current knowledge, the effects on glucose metabolism appear attributable to the different polyphenols content in the study diets. However, a possible role of other known or unknown factors such as trace elements (e.g. copper) cannot be excluded.

A limitation of our study is that participants were characterised by a high cardiometabolic risk; therefore, we do not know whether the same results would also apply to a 'healthy' population, although no evidence is against this hypothesis.

In conclusion, this randomised controlled trial provided evidence that a diet based on different foods and beverages naturally rich in polyphenols improves glucose metabolism in individuals at high risk of diabetes and CVD. Therefore, the best diet for the prevention of diabetes, in addition to the other well-known characteristics, should be rich also in these bioactive components, which are easily found in foods typical of different traditional healthy gastronomic backgrounds (Mediterranean, Nordic, Asian). A diet with higher amounts of fish, rich in LCn3, did not lead to evident benefits on glucose metabolism. Studies with a longer follow-up are needed to evaluate the real impact of these kinds of diet on the incidence of diabetes.

Acknowledgements We are very grateful to L. Patti and L. Di Marino for laboratory assistance; A. Rivieccio for dietary counselling; V. Fogliano (Department of Food Science, Federico II University, Napoli, Italy) for analysing the polyphenol content of some of the foods and beverages; and the patients who participated in this study. Food for the study was kindly supplied by Lavazza, Torino, Italy (coffee); Nestlè, Vevey, Switzerland (chocolate); Zuegg, Verona, Italy (jam); Pompadour Tè, Bolzano, Italy (tea); and Coop Nuovo Cilento, San Mauro Cilento, Salerno, Italy (extra-virgin olive oil).

Parts of this study were presented at the 74th Scientific Sessions of the ADA, San Francisco, California, USA, 13-17 June 2014.

Funding The research leading to these results received funding from the European Community's Seventh Framework Programme FP7/20092012 under grant agreement FP7-KBBE-222639, Etherpaths Project and the 'Ministero Istruzione Università e Ricerca' PRIN 2010-2011 2010JCWWKM. 
Duality of interest statement The authors declare that there is no duality of interest associated with this manuscript.

Contribution statement LB, GA, GR and AAR designed the experiment; LB collected and analysed the data; LB and GA interpreted the data and wrote the manuscript. GP and AT analysed and interpreted the data, and wrote part of the manuscript. GC, CV and EG collected and analysed the data, and wrote part of the manuscript. AG and MV analysed and interpreted the data, and revised the manuscript. CDN, GDP and SC acquired and analysed the data, and revised the manuscript. GR and AAR critically revised the manuscript and contributed to conclusions. AAR is the guarantor of this work and, as such, had full access to all the data in the study and takes responsibility for the integrity of the data and the accuracy of the data analysis. All authors read and approved the final manuscript

\section{References}

1. Tresserra-Rimbau A, Rimm EB, Medina-Remón A, PREDIMED Study Investigators et al (2014) Inverse association between habitual polyphenol intake and incidence of cardiovascular events in the PREDIMED study. Nutr Metab Cardiovasc Dis 24:639-647

2. Mozaffarian D, Wu JH (2011) Omega-3 fatty acids and cardiovascular disease: effects on risk factors, molecular pathways, and clinical events. J Am Coll Cardiol 58:2047-2067

3. Blaak EE, Antoine JM, Benton D et al (2012) Impact of postprandial glycaemia on health and prevention of disease. Obes Rev 13: 923-984

4. Graf BA, Milbury PE, Blumberg JB (2005) Flavonols, flavones, flavanones, and human health: epidemiological evidence. J Med Food 8:281-290

5. Arts IC, Hollman PC (2005) Polyphenols and disease risk in epidemiologic studies. Am J Clin Nutr 81:317S-325S

6. Wedick NM, Pan A, Cassidy A et al (2012) Dietary flavonoid intakes and risk of type 2 diabetes in US men and women. Am J Clin Nutr 95:925-933

7. Babu PV, Liu D, Gilbert ER (2013) Recent advances in understanding the anti-diabetic actions of dietary flavonoids. J Nutr Biochem 24:1777-1789

8. Venables MC, Hulston CJ, Cox HR, Jeukendrup AE (2008) Green tea extract ingestion, fat oxidation, and glucose tolerance in healthy humans. Am J Clin Nutr 87:778-784

9. Chiva-Blanch G, Urpi-Sarda M, Ros E et al (2013) Effects of red wine polyphenols and alcohol on glucose metabolism and the lipid profile: a randomized clinical trial. Clin Nutr 32:200-206

10. van Dijk AE, Olthof MR, Meeuse JC, Seebus E, Heine RJ, van Dam RM (2009) Acute effects of decaffeinated coffee and the major coffee components chlorogenic acid and trigonelline on glucose tolerance. Diabetes Care 32:1023-1025

11. Muley A, Muley P, Shah M (2014) ALA, fatty fish or marine n-3 fatty acids for preventing DM?: a systematic review and meta-analysis. Curr Diabetes Rev 10:158-165

12. Giacco R, Cuomo V, Vessby B, KANWU Study Group et al (2007) Fish oil, insulin sensitivity, insulin secretion and glucose tolerance in healthy people: is there any effect of fish oil supplementation in relation to the type of background diet and habitual dietary intake of n-6 and n-3 fatty acids? Nutr Metab Cardiovasc Dis 17:572-580

13. Maki KC, Lawless AL, Kelley KM, Dicklin MR, Schild AL, Rains TM (2011) Prescription omega-3-acid ethyl esters reduce fasting and postprandial triglycerides and modestly reduce pancreatic beta-cell response in subjects with primary hypertriglyceridemia. Prostaglandins Leukot Essent Fatty Acids 85:143-148
14. Mori TA, Burke V, Puddey IB et al (2000) Purified eicosapentaenoic and docosahexaenoic acids have differential effects on serum lipids and lipoproteins, LDL particle size, glucose, and insulin in mildly hyperlipidemic men. Am J Clin Nutr 71: 1085-1094

15. Sirtori CR, Paoletti R, Mancini M et al (1997) N-3 fatty acids do not lead to an increased diabetic risk in patients with hyperlipidemia and abnormal glucose tolerance. Italian Fish Oil Multicenter Study. Am J Clin Nutr 65:1874-1881

16. Abete I, Parra D, Crujeiras AB, Goyenechea E, Martinez JA (2008) Specific insulin sensitivity and leptin responses to a nutritional treatment of obesity via a combination of energy restriction and fatty fish intake. J Hum Nutr Diet 21:591-600

17. Anderssen SA, Hjermann I, Urdal P, Torjesen PA, Holme I (1996) Improved carbohydrate metabolism after physical training and dietary intervention in individuals with the 'atherothrombogenic syndrome'. Oslo Diet and Exercise Study (ODES). A randomized trial. J Intern Med 240:203-209

18. Zhang J, Wang C, Li L et al (2012) Dietary inclusion of salmon, herring and pompano as oily fish reduces CVD risk markers in dyslipidaemic middle-aged and elderly Chinese women. Br J Nutr 108:1455-1465

19. National Cholesterol Education Program (NCEP) Expert Panel on Detection, Evaluation, and Treatment of High Blood Cholesterol in Adults (Adult Treatment Panel III) (2002) Third Report of the National Cholesterol Education Program (NCEP) Expert Panel on Detection, Evaluation, and Treatment of High Blood Cholesterol in Adults (Adult Treatment Panel III) final report. Circulation 106: 3143-3421

20. Annuzzi G, Bozzetto L, Costabile G et al (2014) Diets naturally rich in polyphenols improve fasting and postprandial dyslipidemia and reduce oxidative stress: a randomized controlled trial. Am J Clin Nutr 99:463-471

21. Palermo M, Pellegrini N, Fogliano V (2014) The effect of cooking on the phytochemical content of vegetables. J Sci Food Agric 94: $1057-1070$

22. Box JD (1983) Investigation of the Folin-Ciocalteu phenol reagent for the determination of polyphenolic substances in natural waters. Water Res 17:511-525

23. USDA (2007) Database for the flavonoid content of selected foods, release 2.1. USDA, Washington

24. Carnovale E, Marletta L (2000) Tabelle di Composizione degli Alimenti- Aggiornamento 2000 Inran - [Food composition database- Update by the National Institute for Food and Nutrition in 2000.]. EDRA, Milan, article in Italian

25. Di Marino L, Griffo E, Maione S, Mirabella M (2011) Active glucagon-like peptide-1 (GLP-1): storage of human plasma and stability over time. Clin Chim Acta 412:1693-1694

26. Pacini G, Mari A (2003) Methods for clinical assessment of insulin sensitivity and beta-cell function. Best Pract Res Clin Endocrinol Metab 17:305-322

27. Stadler M, Pacini G, Petrie J, Luger A, Anderwald C, Investigators RISC (2009) Beta cell (dys)function in non-diabetic offspring of diabetic patients. Diabetologia 52:2435-2444

28. Lankinen M, Kolehmainen M, Jääskeläinen T et al (2014) Effects of whole grain, fish and bilberries on serum metabolic profile and lipid transfer protein activities: a randomized trial (Sysdimet). PLOS ONE 9:e90352

29. Salas-Salvadó J, Bulló M, Babio N, PREDIMED Study Investigators et al (2011) Reduction in the incidence of type 2 diabetes with the Mediterranean diet: results of the PRED IMED-Reus nutrition intervention randomized trial. Diabetes Care 34:14-19

30. Qa'dan F, Verspohl EJ, Nahrstedt A, Petereit F, Matalka KZ (2009) Cinchonain $\mathrm{Ib}$ isolated from Eriobotrya japonica induces insulin secretion in vitro and in vivo. J Ethnopharmacol 124:224-227 
31. Liu IM, Chen WC, Cheng JT (2003) Mediation of beta-endorphin by isoferulic acid to lower plasma glucose in streptozotocininduced diabetic rats. J Pharmacol Exp Ther 307:1196-1204

32. Zunino SJ, Storms DH, Stephensen CB (2007) Diets rich in polyphenols and vitamin A inhibit the development of type I autoimmune diabetes in nonobese diabetic mice. J Nutr 137:1216-1221

33. Scazzocchio $\mathrm{B}$, Varì R, Filesi $\mathrm{C}, \mathrm{D}$ 'Archivio $\mathrm{M}$, Santangelo $\mathrm{C}$, Giovannini $C$ (2011) Cyanidin-3-O- $\beta$-glucoside and protocatechuic acid exert insulin-like effects by upregulating PPAR $\gamma$ activity in human omental adipocytes. Diabetes 60: 2234-2244

34. Xiao C, Giacca A, Carpentier A, Lewis GF (2006) Differential effects of monounsaturated, polyunsaturated and saturated fat ingestion on glucose-stimulated insulin secretion, sensitivity and clearance in overweight and obese, non-diabetic humans. Diabetologia 49:1371-1379
35. Annuzzi G, Rivellese A, Capaldo B et al (1991) A controlled study on the effects of n-3 fatty acids on lipid and glucose metabolism in non-insulin-dependent diabetic patients. Atherosclerosis 87:65-73

36. Newsholme P, Keane D, Welters HJ, Morgan NG (2007) Life and death decisions of the pancreatic beta-cell: the role of fatty acids. Clin Sci (Lond) 112:27-42

37. Giacca A, Xiao C, Oprescu AI, Carpentier AC, Lewis GF (2011) Lipid-induced pancreatic $\beta$-cell dysfunction: focus on in vivo studies. Am J Physiol Endocrinol Metab 300:E255-E262

38. Robertson MD, Jackson KG, Fielding BA, Morgan LM, Williams CM, Frayn KN (2002) Acute ingestion of a meal rich in n-3 polyunsaturated fatty acids results in rapid gastric emptying in humans. Am J Clin Nutr 76:232-238

39. Scalbert A, Williamson G (2000) Dietary intake and bioavailability of polyphenols. J Nutr 130(Suppl):2073S-2085S 